PROCEEDINGS OF THE

AMERICAN MATHEMATICAL SOCIETY

Volume 132, Number 6, Pages 1827-1837

S 0002-9939(03)07249-6

Article electronically published on October 21, 2003

\title{
A PROPERTY OF DUNFORD-PETTIS TYPE IN TOPOLOGICAL GROUPS
}

\author{
E. MARTÍN-PEINADOR AND V. TARIELADZE
}

(Communicated by N. Tomczak-Jaegermann)

\begin{abstract}
The property of Dunford-Pettis for a locally convex space was introduced by Grothendieck in 1953. Since then it has been intensively studied, with especial emphasis in the framework of Banach space theory.

In this paper we define the Bohr sequential continuity property (BSCP) for a topological Abelian group. This notion could be the analogue to the DunfordPettis property in the context of groups. We have picked this name because the Bohr topology of the group and of the dual group plays an important role in the definition. We relate the BSCP with the Schur property, which also admits a natural formulation for Abelian topological groups, and we prove that they are equivalent within the class of separable metrizable locally quasi-convex groups.

For Banach spaces (or for metrizable locally convex spaces), considered in their additive structure, we show that the BSCP lies between the Schur and the Dunford-Pettis properties.
\end{abstract}

\section{PRELIMinaRies}

The aim of this paper is to give an analogue to the Dunford-Pettis property in the context of topological Abelian groups, as explained in Section [3. In this spirit we define in Section 2 the Bohr sequential continuity property, which holds for a class of groups larger than that of Hausdorff locally compact Abelian (LCA) groups. The most satisfactory situation would be, if for any locally convex space $E$, the BSCP were equivalent to the Dunford-Pettis property. The question does not go so smoothly; we have obtained some related results and an insight to properties which so far had only been treated for topological vector spaces and, in fact, they can be extended to topological Abelian groups.

We now introduce notation and relevant facts. Let $G$ be an Abelian topological group; we denote by $\mathcal{N}_{e}(G)$ the set of all neighborhoods of the neutral element $e$. The group of all continuous homomorphisms (also named characters) from $G$ into the torus $\mathbb{T}=\{z \in \mathbb{C}:|z|=1\}$, considered with pointwise multiplication,

\footnotetext{
Received by the editors June 26, 2001 and, in revised form, April 20, 2002 and January 28, 2003.

2000 Mathematics Subject Classification. Primary 22A05; Secondary 46A16.

Key words and phrases. Dunford-Pettis property, Schur property, Bohr topology, dual group, Pontryagin reflexive, locally convex space.

This paper was written while the second author was enjoying a Sabático at the Complutense University of Madrid. The first author was partially supported by D.G.I.C.Y.T. BFM 2000-0804C03-01.
} 
will be denoted by $G^{\wedge}$; the symbol 1 will stand for the character that is equal to one identically. If $\tau$ is a group topology in $G^{\wedge}$, the corresponding topological group will be denoted by $G_{\tau}^{\wedge}$. The dual group of $G$ is defined as $G^{\wedge}$, endowed with the compact-open topology. It will be denoted by $G_{c}^{\wedge}$, while the second dual is $G^{\wedge \wedge}:=\left(G_{c}^{\wedge}\right)^{\wedge}$, again with the corresponding compact open topology. The group $G$ is called reflexive if the canonical homomorphism $\alpha_{G}: G \rightarrow\left(G_{c}^{\wedge}\right)_{c}^{\wedge}$ (defined by $\alpha_{G}(g)(\chi)=\chi(g)$ for every $g \in G$ and every $\left.\chi \in G^{\wedge}\right)$ is a topological isomorphism. If $\alpha_{G}$ is bijective, it is called semireflexive. By the well-known theorem of Pontryagin and van Kampen, every LCA group is reflexive.

We will work in the more general setting of group dualities. The latter were introduced by Varopoulos in 23 and thoroughly studied by us in [10. A group duality $(X, Y)$ consists of an Abelian group $X$ and a subgroup $Y \subset \operatorname{Hom}(X, \mathbb{T})$, where $\operatorname{Hom}(X, \mathbb{T})$ is the set of homomorphisms of the corresponding groups, with pointwise operation. If $Y$ separates the points of $X$, we say that the duality is separating. For a topological group $G$, the pair $\left(G, G^{\wedge}\right)$ is the standard duality. Following the notation of [10], we denote by $\sigma(X, Y)$ the smallest topology in $X$ for which the elements of $Y$ are continuous. Similarly, $\sigma(Y, X)$ will be the coarsest topology in $Y$ making continuous the characters defined as evaluation on the elements of $X$. In other words, $\sigma(Y, X)$ is the topology in $Y$ of pointwise convergence on $X$. For a fixed group duality $(X, Y)$, we denote by $\alpha$ the group homomorphism from $X$ to $\operatorname{Hom}(Y, \mathbb{T})$ defined by the equality $\alpha(x)(\phi)=\phi(x)$, for every $\phi$ of $Y$.

If $G$ is a topological Abelian group, $\sigma\left(G, G^{\wedge}\right)$ is denominated by the Bohr topology of $G$. Notice that $\left(G, \sigma\left(G, G^{\wedge}\right)\right)$ is a precompact topological group [12] 1] We will say that $G$ is dually separated if $G^{\wedge}$ separates the points of $G$. Clearly, $\sigma\left(G, G^{\wedge}\right)$ is Hausdorff if and only if $G$ is dually separated. This occurs, for instance, if $G$ is an LCA group or, in particular, if it is discrete. The group $G_{c}^{\wedge}$ is always dually separated, since $\alpha(G) \subseteq G^{\wedge \wedge}$ separates the points of $G_{c}^{\wedge}$. Evidently, $\sigma\left(G^{\wedge}, G^{\wedge \wedge}\right)$ is the Bohr topology of $G_{c}^{\wedge}$.

For a given duality $(X, Y)$ we have $(X, \sigma(X, Y))^{\wedge}=Y$ and $(Y, \sigma(Y, X))^{\wedge}=$ $\alpha(X)$ (see [10]). Therefore, the topologies $\sigma(X, Y)$ and $\sigma(Y, X)$ can be viewed as the Bohr topologies of $(X, \sigma(X, Y))$ and of $(Y, \sigma(Y, X))$, respectively. Thus, the groups $\left(G, \sigma\left(G, G^{\wedge}\right)\right),\left(G^{\wedge}, \sigma\left(G^{\wedge}, G\right)\right)$ and $\left(G^{\wedge}, \sigma\left(G^{\wedge}, G^{\wedge \wedge}\right)\right)$ are precompact. In particular, for the real line $\mathbb{R}$, the Bohr topology $\sigma\left(\mathbb{R}, \mathbb{R}^{\wedge}\right)$ is strictly coarser than the usual topology. For the additive discrete group of the integers $\mathbb{Z}$, the corresponding Bohr topology $\sigma\left(\mathbb{Z}, \mathbb{Z}^{\wedge}\right)$ is nondiscrete.

The notion of polar set will be used in the sequel. Let $(X, Y)$ be a group duality, and let $A \subset X, B \subset Y$ be nonempty subsets. The polars of $A$ and $B$ respectively are

and

$$
A^{\triangleright}:=\{\phi \in Y: \operatorname{Re}(\phi(x)) \geq 0, \forall x \in A\}
$$

$$
B^{\triangleleft}:=\{x \in X: \operatorname{Re}(\phi(x)) \geq 0, \forall \phi \in B\},
$$

where $R e$ stands for the real part. The subset $A$ is called quasi-convex with respect to $(X, Y)$ if for every $x \in X \backslash A$, there is some $\chi \in A^{\triangleright}$ such that $\operatorname{Re} \chi(x)<0$.

\footnotetext{
${ }^{1}$ The compact Abelian group $\left(\Gamma, \sigma\left(\Gamma, G^{\wedge}\right)\right)$, where $\Gamma=\operatorname{Hom}\left(G^{\wedge}, \mathbb{T}\right)$, is called in the literature the Bohr compactification of $G$. It is not a compactification in the sense of General Topology, unless $G$ is endowed with the Bohr topology. The density of $\alpha_{G}(G)$ in $\left(\Gamma, \sigma\left(\Gamma, G^{\wedge}\right)\right)$ follows from a Stone-Weierstrass type theorem; see [12, Theorem 1.9]. See also [11 for a deep study of the Bohr topology.
} 
The quasi-convex hull of any subset $H \subset X$ is defined as the set $\left(H^{\triangleright}\right)^{\triangleleft}$. A subset $A \subset G$, quasi-convex with respect to $\left(G, G^{\wedge}\right)$, will be simply called quasi-convex. An Abelian topological group $G$ is locally quasi-convex if it has a neighborhood basis of the neutral element $e$, given by quasi-convex sets. The dual $G_{c}^{\wedge}$ of any Abelian topological group $G$ is locally quasi-convex. This implies that any reflexive group is locally quasi-convex. We refer the reader to [1, [2, [10] for more information about reflexive and locally quasi-convex groups.

\section{Continuity of the evaluation mapping $w: G^{\wedge} \times G \rightarrow \mathbb{T}$}

Let $G$ be an Abelian topological group. The evaluation mapping $w: G^{\wedge} \times G \rightarrow \mathbb{T}$ is defined by the formula $w(\phi, x) \mapsto \phi(x), \phi \in G^{\wedge}, x \in G$. A topology $\tau$ in $G^{\wedge}$ is called admissible if $w$ is continuous with respect to the product topology in $G_{\tau}^{\wedge} \times G$. In this section it will become clear that "admissibility" is a very strong condition. On behalf of this, the main property in this paper, introduced in Section 2 is based on sequential continuity of the evaluation.

In a previous work 20, the first author obtained that there are no reflexive groups outside the class of LCA groups, for which the compact open topology is admissible. As we will see next, this fact holds for a class of groups larger than that of reflexive ones. An Abelian topological group $G$ is said to have the quasi-convex compactness property (or briefly, the $q c p$ ) if the quasi-convex hull of every compact subset of $G$ is again compact:

Theorem 1.1. Let $G$ be an Abelian topological group with qcp. If $w: G_{c}^{\wedge} \times G \rightarrow \mathbb{T}$ is continuous, then $G$ is locally compact.

Proof. The continuity of $w$ in $(\mathbf{1}, 0)$ implies we can find neighborhoods $V \in \mathcal{N}_{1}\left(G_{c}^{\wedge}\right)$ and $U \in \mathcal{N}_{0}(G)$, such that

$$
\operatorname{Re} \phi(x) \geq 0, \forall x \in U, \forall \phi \in V .
$$

This gives $U \subset V^{\triangleleft}$. For $V \in \mathcal{N}_{1}\left(G_{c}^{\wedge}\right)$, there exists a compact subset $L$ of $G$ such that $L^{\triangleright} \subset V$ and consequently $U \subset\left(L^{\triangleright}\right)^{\triangleleft}$. By the qcp of $G$, the set $\left(L^{\triangleright}\right)^{\triangleleft}$ is compact and it is a neighborhood of 0 .

Proposition 1.2. Let $G$ be an Abelian topological group, and let $\tau$ be a group topology in $G^{\wedge}$. The following assertions are equivalent:

(a) $w: G_{\tau}^{\wedge} \times G \rightarrow \mathbb{T}$ is continuous.

(b) There exists a Bohr-closed neighborhood $W$ of 0 in $G$ such that $\alpha_{G}(W)$ is equicontinuous on $G_{\tau}^{\wedge}$ and relatively compact in $\left(G_{\tau}^{\wedge}\right)_{c}^{\wedge}$.

$\left(\mathrm{b}^{\prime}\right)$ There exists a Bohr-closed neighborhood $W$ of 0 in $G$ such that $\alpha_{G}(W)$ is equicontinuous on $G_{\tau}^{\wedge}$.

(c) There exists a neighborhood of $\mathbf{1}$ in $G_{\tau}^{\wedge}$ that is equicontinuous and compact in $G_{c}^{\wedge}$.

$\left(\mathrm{c}^{\prime}\right)$ There exists a neighborhood of $\mathbf{1}$ in $G_{\tau}^{\wedge}$ that is equicontinuous.

Proof. (a) $\Rightarrow$ (b), (a) $\Rightarrow$ (c). The continuity of $w$ in $(\mathbf{1}, 0)$ implies that we can find neighborhoods of 1 and $0, V \in \mathcal{N}_{1}\left(G_{\tau}^{\wedge}\right)$ and $U \in \mathcal{N}_{0}(G)$ respectively, such that $V \subset U^{\triangleright}$ and $U \subset V^{\triangleleft}$. Therefore, $U^{\triangleright}$ is a neighborhood of 1 in $G_{\tau}^{\wedge}$. Since $U^{\triangleright}$ is

\footnotetext{
${ }^{2}$ This property, first defined in [5], is the natural analogue of the convex compactness property, well known for locally convex vector spaces. In the same reference it can be seen that a reflexive group has the qcp.
} 
equicontinuous and compact in $G_{c}^{\wedge}$ (see, e.g., [10]), it satisfies the conditions of (c). Since $U \subset V^{\triangleleft}$, it follows that (b) holds for $W:=V^{\triangleleft}$.

(b) $\Rightarrow\left(\mathrm{b}^{\prime}\right)$ and $(\mathrm{c}) \Rightarrow\left(\mathrm{c}^{\prime}\right)$ are trivial.

$\left(\mathrm{c}^{\prime}\right) \Rightarrow(\mathrm{a})$. In order to prove the continuity of $w$ at $(\mathbf{1}, 0)$, take $L \in \mathcal{N}_{1}(\mathbb{T})$. If $V \in \mathcal{N}_{\mathbf{1}}\left(G_{\tau}^{\wedge}\right)$ is equicontinuous, there exists $U \in \mathcal{N}_{0}(G)$ such that $w(U \times V) \subseteq L$. The continuity of $w$ at any point $(\phi, x) \in G^{\wedge} \times G$ follows easily from the continuity at $(\mathbf{1}, 0)$.

The proof of $\left(b^{\prime}\right) \Rightarrow(a)$ is similar.

Corollary 1.3. Let $\tau$ be a Hausdorff group topology in $G^{\wedge}$. The following assertions hold:

(a) If $w$ is $\tau \times \sigma\left(G, G^{\wedge}\right)$-continuous, then $\tau$ is discrete.

(b) If $\tau=\sigma\left(G^{\wedge}, G^{\wedge \wedge}\right)$, or $\tau=\sigma\left(G^{\wedge}, \Gamma\right)$ with $\Gamma=\operatorname{Hom}\left(G^{\wedge}, \mathbb{T}\right)$, and $w$ is $\tau \times \sigma\left(G, G^{\wedge}\right)$-continuous, then $G^{\wedge}$ is finite. Also, $G$ is finite, provided it is dually separated.

Proof. (a) Since $\left(G, \sigma\left(G, G^{\wedge}\right)\right)^{\wedge}=G^{\wedge}$, we can apply (a) $\Rightarrow\left(\mathrm{c}^{\prime}\right)$ of Proposition 1.2 to the group $\left(G, \sigma\left(G, G^{\wedge}\right)\right)$ and obtain that $G_{\tau}^{\wedge}$ has a $\sigma\left(G, G^{\wedge}\right)$-equicontinuous neighborhood of $\mathbf{1}$, say $V$. This implies that $V \subset\left(B^{\triangleleft}\right)^{\triangleright}$ for a finite nonempty subset $B$ of $G^{\wedge}$. According to [1 (7.11)] the set $\left(B^{\triangleleft}\right)^{\triangleright}$ is finite. Therefore, the group $G_{\tau}^{\wedge}$ has a finite neighborhood of $\mathbf{1}$. Since $\tau$ is a Hausdorff group topology, it is discrete.

(b) follows from (a) together with the fact that $G_{\tau}^{\wedge}$ is precompact and Hausdorff.

\section{The Bohr SEQUential CONTINUITy PROPERTY}

We will say that an Abelian topological group $G$ has the Bohr sequential continuity property or, for short, the BSCP if the evaluation mapping

$$
w:\left(G^{\wedge}, \sigma\left(G^{\wedge}, G^{\wedge \wedge}\right)\right) \times\left(G, \sigma\left(G, G^{\wedge}\right)\right) \rightarrow \mathbb{T}
$$

is sequentially continuous with respect to the product topology.

The existence of nonfinite topological groups with the BSCP is not clear just from the definition. In order to provide such examples, we first present some auxiliary notions and results.

Recall that an Abelian topological group $G$ is said to have: 1) the Schur property if any $\sigma\left(G, G^{\wedge}\right)$-convergent sequence is also convergent in the original topology of $G$, and 2) the Glicksberg property or $G$ respects compactness if any $\sigma\left(G, G^{\wedge}\right)$-compact subset of $G$ is also compact in the original topology of $G$. By a well-known theorem of Glicksberg, every LCA group respects compactness. Observe that if $G$ has the Schur or the Glicksberg property, the same holds for any of its subgroups. It is standard to check that a dually separated group $G$ with the Glicksberg property has the Schur property too.

In [10], we called g-barrelled those Abelian topological groups $G$ for which the $\sigma\left(G^{\wedge}, G\right)$-compact subsets of $G^{\wedge}$ are equicontinuous. We now define a sort of sequential version of $g$-barrelledness. An Abelian topological group $G$ is sequentially barrelled if every $\sigma\left(G^{\wedge}, G\right)$-convergent sequence in $G^{\wedge}$ is equicontinuous. Evidently, any $g$-barrelled group is sequentially barrelled (the converse is not true in general). The next assertion shows that the class of sequentially barrelled groups includes Baire groups; its proof follows from [10, (1.4)]. 
Proposition 2.1. Let $G$ be an Abelian topological group that is a Baire space. Then, $G$ is sequentially barrelled. Moreover, $\left(G^{\wedge}, \sigma\left(G^{\wedge}, G\right)\right)$ is sequentially complete.

Remark 2.2. We do not know if every Baire group is $g$-barrelled. Any of the following three conditions are sufficient for a group $G$ to be $g$-barrelled (cf. [10]):

(1) $G$ is metrizable and all of its closed subgroups are Baire spaces,

(2) $G$ is Čech-complete,

(3) $G$ is separable Baire.

LCA groups are Čech-complete and, therefore, $g$-barrelled.

Proposition 2.3. For an Abelian topological group $G$, the following assertions hold:

(a) If $G$ is sequentially barrelled, then $G_{c}^{\wedge}$ has the Schur property.

$\left(\mathrm{a}^{\prime}\right)$ If $G_{c}^{\wedge}$ is sequentially barrelled and $\alpha_{G}$ is a topological embedding, then $G$ has the Schur property.

(b) If $G$ is g-barrelled, then $G_{c}^{\wedge}$ has the Glicksberg property.

$\left(\mathrm{b}^{\prime}\right)$ If $G_{c}^{\wedge}$ is g-barrelled and $\alpha_{G}$ is a topological embedding, then $G$ has the Glicksberg property.

(c) If $G$ is metrizable, then $G_{c}^{\wedge}$ has the Glicksberg property 3

Proof. (a) Let $\left\{\phi_{n}: n \in \mathbb{N}\right\}$ be a sequence in $G^{\wedge}$ that converges to 1 in $\sigma\left(G^{\wedge}, G^{\wedge \wedge}\right)$. Clearly, it also converges in $\sigma\left(G^{\wedge}, G\right)$. Since $G$ is sequentially barrelled, $\left\{\phi_{n}: n \in\right.$ $\mathbb{N}\}$ is equicontinuous. This implies that $\left\{\phi_{n}: n \in \mathbb{N}\right\}$ converges to 1 in $G_{c}^{\wedge}$.

$\left(a^{\prime}\right)$ follows from (a) together with the observation that the Schur property is hereditary. The proofs of $(\mathrm{b})$ and $\left(\mathrm{b}^{\prime}\right)$ are similar.

(c) Let $G^{\prime}$ be the completion of $G$. Then $G^{\wedge}=G^{\wedge}$ algebraically. Since $G$ is metrizable, according to [9] Theorem 2], we conclude that $G_{c}^{\wedge}=G^{\prime \wedge}$ also. Now let $B \subset G_{c}^{\wedge}$ be a $\sigma\left(G^{\wedge}, G^{\wedge \wedge}\right)$-compact set; it is compact also in $\sigma\left(G^{\prime \wedge}, G^{\prime \wedge}\right)$. Since $G^{\prime}$ is complete metrizable, it is $g$-barrelled (see Remark [2.2. (1)) and from (b) we get that $B$ is compact in ${G^{\prime}}_{c}^{\wedge}$, and hence in $G_{c}^{\wedge}$.

The next proposition provides nontrivial examples of groups with the BSCP; for the definition of nuclear groups, mentioned in item (e), see [2].

Proposition 2.4. Let $G$ be an Abelian topological group. The following assertions hold:

(a) If $G$ and $G_{c}^{\wedge}$ have the Schur property, then $G$ has the BSCP.

(b) If $G$ has the Schur property and is sequentially barrelled, then $G$ has the $B S C P$.

(c) If $G$ is metrizable and has the Schur property, then $G$ has the BSCP.

(d) If $G$ is locally compact and Hausdorff, then $G$ has the BSCP.

(e) If $G$ is metrizable nuclear or Čech-complete nuclear, then $G$ has the BSCP.

Proof. From the definition of the compact open topology it is straightforward that the evaluation $w: G_{c}^{\wedge} \times G \rightarrow \mathbb{T}$ is sequentially continuous. This fact immediately proves (a), and together with Proposition 2.3 implies (b). The proof of (c) follows from (a) and Proposition 2.3 (c). For (d) apply first Proposition 2.3 (b), (b') to obtain that $G$ and $G_{c}^{\wedge}$ have the Schur property. Then item (a) finishes the proof.

\footnotetext{
${ }^{3}$ We had this result for metrizable topological vector spaces in an earlier version of the present paper. Its validity for this general setting was obtained in collaboration with María Jesús Chasco.
} 
Finally, note that any nuclear group respects compactness [3], and hence it has the Schur property. If $G$ is metrizable, (e) follows from (c). For a Čech-complete nuclear group, its dual is again nuclear [1, (20.36)]; now since $G$ and $G_{c}^{\wedge}$ have the Schur property, item (a) applies.

Remark 2.5. (i) A nuclear group may not have the BSCP. An example could be $G=l^{2}$ endowed with its Bohr topology. The same example shows that sequential barrelledness cannot be removed from (b) of the previous proposition.

(ii) The rational numbers with their natural topology constitute an example of a metrizable nuclear group (thus, with BSCP) that is not sequentially barrelled. In fact, by means of the exponential mapping we identify a real number with a character on $\mathbb{Q}$. The sequence $\left\{x_{n}=n !: n \in \mathbb{N}\right\} \subset \mathbb{R}$ converges to the character $\mathbf{1}$ in $\sigma\left(\mathbb{Q}^{\wedge}, \mathbb{Q}\right)$, but it is not equicontinuous. The same example shows that (c) is not a consequence of $(b)$.

We study now how the BSCP is transmitted to the dual. Whether semireflexivity can be removed in the next proposition is not known to the authors.

Proposition 2.6. Let $G$ be an Abelian topological group. The following assertions hold:

(a) If $G$ is semireflexive and has the BSCP, then $G_{c}^{\wedge}$ has the BSCP.

(b) If $\alpha_{G}: G \rightarrow\left(G_{c}^{\wedge}\right)_{c}^{\wedge}$ is continuous and $G_{c}^{\wedge}$ has the BSCP, then $G$ also has the BSCP. In particular, if $G$ is either metrizable or g-barrelled and $G_{c}^{\wedge}$ has the BSCP, then $G$ has the BSCP.

(c) If $G$ is reflexive, then $G$ has the BSCP if and only if $G_{c}^{\wedge}$ has the BSCP.

Proof. (a) can be derived from the fact that $\alpha_{G}\left(x_{n}\right) \rightarrow \mathbf{1}$ in $\sigma\left(G^{\wedge \wedge}, G^{\wedge \wedge \wedge}\right)$ implies that $x_{n} \rightarrow 0$ in $\sigma\left(G, G^{\wedge}\right)$, for any sequence $\left\{x_{n}: n \in \mathbb{N}\right\} \subset G$.

(b) and (c) are easily proved.

The following formulation of the BSCP in terms of uniform convergence might have some interest of its own. It allows us to prove that, under suitable conditions, the BSCP implies the Schur property (Proposition 2.8).

Proposition 2.7. For an Abelian topological group $G$, the following statements are equivalent:

(a) G has the BSCP.

(b) Any Bohr convergent sequence $\left\{x_{n}: n \in \mathbb{N}\right\} \subset G$ converges uniformly on any $\sigma\left(G^{\wedge}, G^{\wedge \wedge}\right)$-sequentially compact subset $K$ of $G^{\wedge}$.

(c) Any $\sigma\left(G^{\wedge}, G^{\wedge \wedge}\right)$-convergent sequence $\left\{\phi_{n}: n \in \mathbb{N}\right\} \subset G^{\wedge}$ converges uniformly on $\sigma\left(G, G^{\wedge}\right)$-sequentially compact subsets of $G$.

Proof. (a) $\Rightarrow$ (b) Suppose $x_{n} \rightarrow 0$ in $\sigma\left(G, G^{\wedge}\right)$. If

$$
\sup _{\phi \in K}\left|1-\phi\left(x_{n}\right)\right| \rightarrow 0
$$

were not true, there would exist $\epsilon>0$ (passing to a subsequence if necessary), such that

$$
\sup _{\phi \in K}\left|1-\phi\left(x_{n}\right)\right|>\epsilon, n=1,2, \ldots
$$

Then, for some sequence $\left\{\phi_{n}: n \in \mathbb{N}\right\}$ in $K$,

$$
\left|1-w\left(\phi_{n}, x_{n}\right)\right|=\left|1-\phi_{n}\left(x_{n}\right)\right|>\epsilon, n=1,2, \ldots
$$


Since $K$ is $\sigma\left(G^{\wedge}, G^{\wedge \wedge}\right)$-sequentially compact, there is a subsequence $\left(\phi_{n_{j}}\right)$ of $\left(\phi_{n}\right)$ and a character $\phi \in K$ such that $\phi_{n_{j}} \rightarrow \phi$. By the BSCP of $G, w\left(\phi_{n_{j}}, x_{n_{j}}\right) \rightarrow 1$. This contradicts $(*)$.

(b) $\Rightarrow$ (a) Take $x_{n} \rightarrow 0$ in the Bohr topology of $G$ and $\varphi_{n} \rightarrow \mathbf{1}$ in $\sigma\left(G^{\wedge}, G^{\wedge \wedge}\right)$. An easy argument shows that the compact set $K:=\left\{\varphi_{n}: n \in \mathbb{N}\right\} \cup\{\mathbf{1}\}$ is also sequentially compact in $\sigma\left(G^{\wedge}, G^{\wedge \wedge}\right)$. Therefore, by (b), $\sup _{\varphi \in K}\left|1-\varphi\left(x_{n}\right)\right| \rightarrow 0$. Thus $\left|1-\varphi_{n}\left(x_{n}\right)\right| \rightarrow 0$ and $\varphi_{n}\left(x_{n}\right) \rightarrow 1$.

(a) $\Leftrightarrow$ (c) can be proved similarly.

Proposition 2.8. Let $G$ be an Abelian topological group with the BSCP. Then $G$ has the Schur property, provided one of the following conditions holds:

(1) $G$ is locally quasi-convex and the equicontinuous $\sigma\left(G^{\wedge}, G\right)$-compact subsets of $G^{\wedge}$ are $\sigma\left(G^{\wedge}, G\right)$-sequentially compact.

(2) $G$ is locally quasi-convex and separable.

(3) $G$ is separable and $\alpha_{G}$ is a topological embedding.

(4) $G$ is a separable reflexive group.

Proof. Let us prove that if (1) holds, then $G$ has the Schur property. The rest of the proof follows from the observation that $(4) \Rightarrow(3) \Rightarrow(2) \Rightarrow(1)$.

Take a sequence $\left\{x_{n}: n \in \mathbb{N}\right\} \subset G$ convergent to 0 in $\sigma\left(G, G^{\wedge}\right)$. Since $G$ is locally quasi-convex, in order to show that $x_{n} \rightarrow 0$, it is sufficient to see that it is eventually contained in any quasi-convex neighborhood of 0 . Thus, let $U \in \mathcal{N}_{0}(G)$ be such that $U=\left(U^{\triangleright}\right)^{\triangleleft}$. The set $U^{\triangleright}$ is $\sigma\left(G^{\wedge}, G\right)$-compact and equicontinuous (cf. [10, $1.1(\mathrm{c})])$. By $(1), U^{\triangleright}$ is $\sigma\left(G^{\wedge}, G\right)$-sequentially compact and, due to the fact that the compact-open topology coincides with $\sigma\left(G^{\wedge}, G\right)$ on equicontinuous subsets, $U^{\triangleright}$ is also a sequentially compact subset of $G_{c}^{\wedge}$. Consequently, it is $\sigma\left(G^{\wedge}, G^{\wedge \wedge}\right)$ sequentially compact. From Proposition 2.7 we obtain

$$
\sup _{\phi \in U^{\triangleright}}\left|1-\phi\left(x_{n}\right)\right| \rightarrow 0 .
$$

Thus, $x_{n} \in\left(U^{\triangleright}\right)^{\triangleleft}=U$ for sufficiently large $n$, which concludes the proof.

Within the class of separable metrizable locally quasi-convex groups, there is even equivalence between the BSCP and the Schur property, as shown in Theorem 2.9] whose proof derives from Propositions 2.4(c) and 2.8. Observe that separability cannot be removed from it. In fact, in Proposition 3.4 we give an example of a complete metrizable reflexive group with the BSCP that does not have the Schur property.

Theorem 2.9. Let $G$ be a separable metrizable locally quasi-convex group or, in particular, a separable metrizable reflexive group. Then $G$ has the BSCP if and only if it has the Schur property.

Corollary 2.10. Let $G$ be a Frechet space that is either separable or reflexive in the ordinary sense. Then $G$, considered as a group, has the BSCP if and only if it has the Schur property.

Proof. Note that a locally convex space considered as a group is locally quasi-convex [2, (2.4)]. Thus, if $G$ is separable, the equivalence follows from Theorem [2.9. Under the assumption of ordinary reflexivity (without separability), taking into account that $\sigma\left(G^{\wedge}, G\right)$-compact subsets of $G^{\wedge}$ are also $\sigma\left(G^{\wedge}, G\right)$-sequentially compact [6] Theorem 11 (ii)], the proof follows from Propositions [2.8 and [2.4(c). 
The above results have a "Functional Analysis" flavour. In fact, they show that notions like barrelledness, the Schur property, etc., widely known in the framework of topological vector spaces, can be successfully studied within the class of topological groups.

\section{The Dunford-Pettis property versus the BSCP FOR LOCALLY CONVEX SPACES}

In the previous section we defined the BSCP in an attempt to extend the Dunford-Pettis property from locally convex spaces to Abelian topological groups. However, a total coincidence of the two properties cannot be expected, since dual groups are endowed with the compact open topology while dual vector spaces are endowed with the topology of uniform convergence on bounded subsets. Thus, the bidual space of a locally convex space is not, in general, algebraically isomorphic to its bidual group, obtained by considering only its additive structure.

In order to make our ideas precise, after some standard notation for the vector space setting, we shall study the Schur, the BSCP and the Glicksberg properties for the very special class of Abelian topological groups formed by the locally convex spaces.

Let $E$ be a locally convex space. The dual space of $E$ is the vector space of continuous linear functionals $E^{*}$, endowed with the topology of uniform convergence on bounded subsets of $E$; it will be denoted by $E_{\beta}^{*}$ (or simply $E^{*}$, since this is the most natural topology for the dual of a topological vector space). The set $E^{*}$ endowed with the compact open topology, $E_{c}^{*}$, will be called the Pontryagin dual space of $E$ in resemblance to the group theory.

We put $E^{* *}:=\left(E_{\beta}^{*}\right)^{*}$; it is clear that $\left(E_{c}^{*}\right)^{*}$ is a vector subspace of $E^{* *}$, which in general may be proper. The evaluation mapping $e: E^{*} \times E \rightarrow \mathbb{R}$ is defined by $e\left(x^{*}, x\right)=x^{*}(x)$. Recall that $E$ is said to be reflexive if the mapping $x \rightarrow e(\cdot, x)$ from $E$ to $\left(E_{\beta}^{*}\right)_{\beta}^{*}$ is a homeomorphism. If it is only bijective, $E$ is called semireflexive. Analogously, $E$ is Pontryagin reflexive if the mapping $x \rightarrow e(\cdot, x)$ from $E$ to $\left(E_{c}^{*}\right)_{c}^{*}$ is a homeomorphism, and Pontryagin semireflexive if it is a bijection.

The following well-known facts were established in [22]:

- $E^{\wedge}=\left\{\exp \left(2 \pi i x^{*}\right): x^{*} \in E^{*}\right\}$.

- The mapping $x^{*} \rightarrow \exp \left(2 \pi i x^{*}\right)$ is a topological group isomorphism between $E_{c}^{*}$ and $E_{c}^{\wedge}$ that preserves the equicontinuous subsets.

- The space $E$ is Pontryagin reflexive (or semireflexive) iff it is reflexive (or semireflexive) as a group.

The symbols $\sigma\left(E, E^{*}\right), \sigma\left(E^{*}, E\right), \sigma\left(E^{*}, E^{* *}\right)$ and $\sigma\left(E^{*},\left(E_{c}^{*}\right)^{*}\right)$ will have their usual meaning; we denominate them weak or weak-star topologies, as is done in the theory of locally convex spaces.

Observe that, whenever $E^{*} \neq\{0\}$, the Bohr topology $\sigma\left(E, E^{\wedge}\right)$ is strictly coarser than the weak topology $\sigma\left(E, E^{*}\right)$ and the same is true for the Bohr topology and the weak topology of the locally convex space $E_{c}^{*}$. However, the topological vector space $\left(E, \sigma\left(E, E^{*}\right)\right)$ and the topological group $\left(E, \sigma\left(E, E^{\wedge}\right)\right)$ have the same convergent sequences and the same compact subsets 21]. A direct proof of this fact can also be derived from the assertion that $\mathbb{R}$, as a group, has the Schur and the Glicksberg properties. Next, we transcribe our definitions of the Schur, the Glicksberg and the $\mathrm{BSCP}$ to the language of locally convex spaces. 
Lemma 3.1. Let $E$ be a locally convex space. The following assertions hold:

(a) E has the Schur property as a topological group iff any weakly convergent sequence of elements of $E$ converges in the original topology of $E$.

(b) E has the Glicksberg property as a topological group iff any weakly compact subset of $E$ is compact in the original topology of $E$.

(c) $E$ has the BSCP iff the mapping e: $\left(E^{*}, \sigma\left(E^{*},\left(E_{c}^{*}\right)^{*}\right)\right) \times\left(E, \sigma\left(E, E^{*}\right)\right) \rightarrow \mathbb{R}$ is sequentially continuous.

A Hausdorff locally convex space $E$ is said to have the Dunford-Pettis property if $e:\left(E^{*}, \sigma\left(E^{*}, E^{* *}\right)\right) \times\left(E, \sigma\left(E, E^{*}\right)\right) \rightarrow \mathbb{R}$ is sequentially continuous. Such a formulation is commonly accepted in Banach space theory, where this property has been widely studied; a very nice survey on the topic is given in [13. Next, we relate it to the previously studied properties.

Proposition 3.2. Let $E$ be a locally convex space. Then:

(a) If $E$ has the BSCP, it also has the Dunford-Pettis property. In particular, if $E$ is metrizable with the Schur property, it also has the Dunford-Pettis property.

(b) If $E_{\beta}^{*}$ has the Schur property, then $E$ has the Dunford-Pettis property. Thus, $c_{0}$ has the Dunford-Pettis property.

$\left(\mathrm{b}^{\prime}\right)$ If $E$ is metrizable and $E_{\beta}^{*}$ has the Dunford-Pettis property, then $E$ has the Dunford-Pettis property.

(c) A Banach space may have the Dunford-Pettis property and fail to have the $B S C P$. The space $c_{o}$ is such an example.

Proof. (a) follows from Lemma 3.1(c), if we note that $\left(E_{c}^{*}\right)^{*}$ is included in $\left(E_{\beta}^{*}\right)^{*}$, and hence $\sigma\left(E^{*},\left(E_{c}^{*}\right)^{*}\right) \subset \sigma\left(E^{*}, E^{* *}\right)$.

(b) Take $x_{n}^{*} \stackrel{\sigma\left(E^{*}, E^{* *}\right)}{\longrightarrow} 0$ and $x_{n} \stackrel{\sigma\left(E, E^{*}\right)}{\longrightarrow} 0$. Under the assumption that $E_{\beta}^{*}$ has the Schur property, we obtain that $x_{n}^{*} \rightarrow 0$ in $E_{\beta}^{*}$. Therefore, $x_{n}^{*} \rightarrow 0$ uniformly on bounded subsets of $E$. Since $\left\{x_{n}: n \in \mathbb{N}\right\}$ is bounded, $x_{n}^{*}\left(x_{n}\right) \rightarrow 0$ in $\mathbb{R}$.

For the last statement, note that $l_{1}$ is the strong dual of $c_{0}$, and it has the Schur property.

$\left(\mathrm{b}^{\prime}\right)$ Since $E$ is metrizable, the mapping $x \rightarrow e(\cdot, x)$ is continuous from $E$ to $\left(E_{\beta}^{*}\right)_{\beta}^{*}$. The rest follows easily.

(c) follows from the second part of (b) and Corollary 2.10, since $c_{0}$ does not have the Schur property.

Remark 3.3. (i) In 15] it was proved that the Banach space $L_{1}(\Omega, \mathcal{A}, \mu)$, where $(\Omega, \mathcal{A}, \mu)$ is an arbitrary positive measure space, has the Dunford-Pettis property. The space $C(M)$ of continuous functions on a compact Hausdorff space $M$ also has the Dunford-Pettis property, 18. Therefore, $l_{\infty}$ has the Dunford-Pettis property, since $l_{\infty}$ can be identified with $C(\beta \mathbb{N})$, where $\beta \mathbb{N}$ is the Stone-Čech compactification of the natural numbers.

(ii) The converse to Proposition $3.2\left(\mathrm{~b}^{\prime}\right)$ does not hold. In fact, $l_{1}$ has a closed vector subspace $E$ such that $E_{\beta}^{*}$ does not have the Dunford-Pettis property (see [13, pp. 20-23]). Thus, it might even happen that a separable Banach space has the Schur property (hence, also Dunford-Pettis), and its strong dual does not have the Dunford-Pettis property.

(iii) The Schur property for a Banach space has been characterized in terms of the Bohr compactification of its underlying group in [16]. The Dunford-Pettis 
property for the vector-valued versions of $c_{0}, l_{1}, C(M)$ is investigated, for instance, in [4, [7, 8].

In general, the BSCP does not imply the Schur property, as we prove below.

Proposition 3.4. Let $E$ be $l_{\infty}$ with its usual norm topology. Then, $E$ has the $B S C P$ and fails to have the Schur property.

Proof. Take $\left\{x_{n}: n \in \mathbb{N}\right\} \subset E$ and $\left\{x_{n}^{*}: n \in \mathbb{N}\right\} \subset E^{*}$ such that $x_{n} \rightarrow 0$ in $\sigma\left(E, E^{*}\right)$ and $x_{n}^{*} \rightarrow 0$ in $\sigma\left(E^{*},\left(E_{c}^{*}\right)^{*}\right)$. Evidently, $x_{n}^{*} \rightarrow 0$ in $\sigma\left(E^{*}, E\right)$. By a result of Grothendieck (see [14, Theorem 15, p. 103]), this implies that $x_{n}^{*} \rightarrow 0$ in $\sigma\left(E^{*}, E^{* *}\right)$. Since $E$ has the Dunford-Pettis property (Remark $3.3(\mathrm{i})$ ), we obtain that $x_{n}^{*}\left(x_{n}\right) \rightarrow$ 0 . Consequently, the mapping $e:\left(E^{*}, \sigma\left(E^{*},\left(E_{c}^{*}\right)^{*}\right)\right) \times\left(E, \sigma\left(E, E^{*}\right)\right) \rightarrow \mathbb{R}$ is sequentially continuous and $E$ has the BSCP.

On the other hand, $E$ does not have the Schur property; in fact, it contains a closed vector subspace without it, namely $c_{0}$.

We can thus conclude that the BSCP, introduced by us in the framework of Abelian topological groups, defines a new notion in the realm of Banach spaces, distinct from the Schur and the Dunford-Pettis properties.

\section{ACKNowledgement}

The authors are very indebted to the referee, for his (or her) careful reading of the manuscript. His numerous corrections have led us to a substantially improved version.

\section{REFERENCES}

1. Aussenhofer, L., Contributions to the Duality Theory of Abelian Topological Groups and to the Theory of Nuclear Groups. Dissertationes Mathematicae 384 (1999), 113 pp. MR 2001c:43003

2. Banaszczyk, W., Additive Subgroups of Topological Vector Spaces, Lecture Notes in Mathematics 1466, Springer-Verlag, Berlin, Heidelberg, New York, 1991. MR 93b:46005

3. Banaszczyk, W., and E. Martín-Peinador, The Glicksberg theorem on weakly compact sets for nuclear groups, Annals of the New York Academy of Sciences 788 (1996), 34-39. MR 99e:22001

4. Bombal, F., and I. Villanueva, On the Dunford-Pettis property of the tensor products of $C(K)$ spaces, Proc. Amer. Math. Soc. 129 (2001), 1359-1363. MR 2001h:46024

5. Bruguera, M., Grupos topológicos y grupos de convergencia: estudio de la dualidad de Pontryagin, Doctoral Dissertation, 1999.

6. Cascales, B., and J. Orihuela, On compactness in locally convex spaces, Math. Z. 195 (1987), 365-381. MR 88i:46021

7. Cembranos, P., The hereditary Dunford-Pettis property on $C(K, E)$, Illinois J. Math. 31 (1987), 365-373. MR 88g:46028

8. Cembranos, P., The hereditary Dunford-Pettis property for $l_{1}(E)$, Proc. Amer. Math. Soc. 108 (1990), 947-950. MR 90i:46019

9. Chasco, M. J., Pontryagin duality for metrizable groups, Archiv der Math. 70 (1998), 22-28. MR 98k:22003

10. Chasco, M. J., E. Martín-Peinador, and V. Tarieladze, On Mackey topology for groups, Studia Math. 132 (1999), 257-284. MR 2000b:46003

11. Comfort, W. W., S. Hernández, and F. J. Trigos-Arrieta, Relating a locally compact Abelian group to its Bohr compactification, Advances in Math. 120 (1996), 322-344. MR 97k:22005

12. Comfort, W. W., and K. A. Ross, Topologies induced by groups of characters, Fundamenta Math. 55 (1964), 283-291. MR 30:183

13. Diestel, J., A survey of results related to the Dunford-Pettis property, Contemporary Mathematics 2 (1980), 15-60. MR 82i:46023 
14. Diestel, J., Sequences and Series in Banach Spaces, Graduate Texts in Mathematics 92, Springer-Verlag, New York, 1984. MR 85i:46020

15. Dunford, N., and B. J. Pettis, Linear operations on summable functions, Trans. Amer. Math. Soc. 47 (1940), 323-392. MR 1:338b

16. Galindo, J., S. Hernández, and S. Macario, A characterization of the Schur property by means of the Bohr topology, Topology and Appl. 97 (1999), 99-108. MR 2000k:22002

17. Glicksberg, I., Uniform boundedness for groups, Canadian Journal of Math. 14 (1962), 269276. MR 27:5856

18. Grothendieck, A., Sur les applications linéaires faiblement compactes d'espaces du type $C(K)$, Canad. J. Math. 5 (1953), 129-173. MR 15:438b

19. Hewitt, E., and K. A. Ross, Abstract Harmonic Analysis I, Die Grundlehren der Mathematischen Wissenschaften 115, Springer-Verlag, Berlin, 1963. MR 28:158

20. Martín-Peinador, E., A reflexive admissible topological group must be locally compact, Proc. Amer. Math. Soc. 123 (1995), 3563-3566. MR 96a:22002

21. Remus, D., and F. J. Trigos-Arrieta, Abelian groups which satisfy Pontryagin duality need not respect compactness, Proc. Amer. Math. Soc. 117 (4) (1993), 1195-1200. MR 93e:22009

22. Smith, M. F., The Pontryagin duality theorem in linear spaces, Ann. Math. 56 No. 2 (1952), 248-253. MR 14:183a

23. Varopoulos, N. T., Studies in harmonic analysis, Proc. Cambridge Philos. Soc. 60 (1964), 465-516. MR 29:1284

Departamento de Geometría y Topología, Universidad Complutense de Madrid, Av. Complutense S/N, Madrid 28040, España

E-mail address: peinador@mat.ucm.es

Muskhelishvili Institute of Computational Mathematics, Georgian Academy of Sciences, Akuri str. 8. Tbilisi 93, Georgia

E-mail address: tar@gw.acnet.ge 